\title{
LORENTZ-SHAPED COMET DUST TRAIL CROSS SECTION FROM NEW HYBRID VISUAL AND VIDEO METEOR COUNTING TECHNIQUE - IMPLICATIONS FOR FUTURE LEONID STORM ENCOUNTERS
}

\author{
PETER JENNISKENS, CHRIS CRAWFORD ${ }^{1}$, STEVEN J. BUTOW, \\ AND DAVID NUGENT \\ SETI Institute, NASA/Ames Research Center, Mail Stop 239-4, Moffett Field, CA \\ 94035, USA; ') 2349 Sterling Creek Road, Jacksonville, OR 97530, USA \\ E-mail:pjenniskens@mail.arc.nasa.gov \\ MIKE KOOP, DAVID HOLMAN, AND JANE HOUSTON \\ California Meteor Society, 1037 Wunderlich Drive, San Jose, CA 95129-3159, USA
}

KLAAS JOBSE
Dutch Meteor Society, Lederkarper 4, 2318 NB Leiden, The Netherlands

GARY KRONK

American Meteor Society, North American Meteor Network, 1117 Troy-O'fallon Road, Troy, Il 62294, USA

and

KELLY BEATTY

Sky \& Telescope, 49 Bay State Road, Cambridge, MA 02138-1200, USA

(Received 4 June 2000; Accepted 18 August 2000)

\begin{abstract}
A new hybrid technique of visual and video meteor observations was developed to provide high precision near real-time flux measurements for satellite operators from airborne platforms. A total of 33,000 Leonids, recorded on video during the 1999 Leonid storm, were watched by a team of visual observers using a video head display and an automatic counting tool. The counts reveal that the activity profile of the Leonid storm is a Lorentz profile. By assuming a radial profile for the dust trail that is also a Lorentzian, we make predictions for future encounters. If that assumption is correct, we passed $0.0003 \mathrm{AU}$ deeper into the 1899 trailet than expected during the storm of 1999 and future encounters with the 1866 trailet will be less intense than predicted elsewhere.
\end{abstract}

Keywords: Comet, comet: 55P/Tempel-Tuttle, dust trail, flux, Leonids 1999, Lorentz profile, meteor, meteor storm, predictions, satellite impact hazard, observing techniques 
of each viewing session. This permitted the computer to identify the starting and ending times of each viewing session, and determine which observer was watching from what camera at all times. Rotating the observer/camera pairings enabled calculation of individual observer and camera coefficients of perception from systematic differences in the counts.

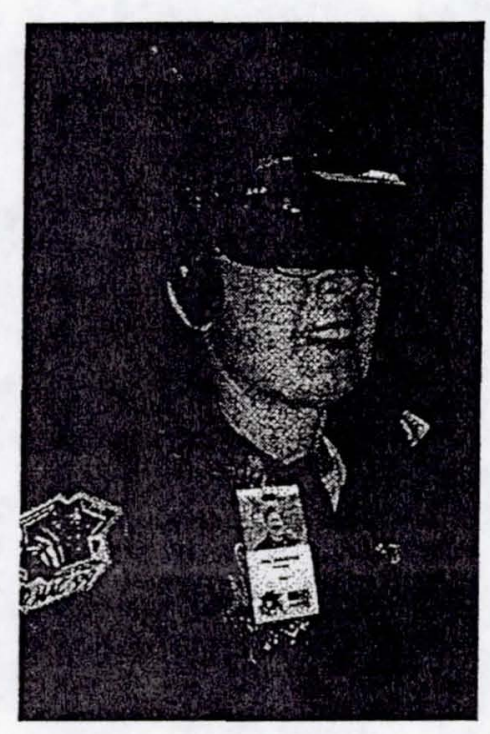

Figure 1. Observer Jane Houston with video head display.

During the 1999 Leonid meteor storm, ARIA flew from the UK to Israel, from Israel to the Azores, and from the Azores to Florida on three consecutive nights. The peak of the storm occurred while enroute from Greece to Italy. Near-real time flux measurements were automatically transferred to a communication station on-board the aircraft, where the counts were sent to NASA/Ames Research Center by e-mail, telephone or direct internet access using INMARSAT satellite telephone lines. From NASA/ARC, the counts were further distributed to operation centers, such as the NASA and USAF sponsored LEOC at Marshall Space Flight Center and ESA's orbital debris center at ESOC, Darmstadt.

Shortly after the mission, several observers gathered at NASA/Ames Research Center to view, in the same manner, the video tapes that were recorded by four similar intensified cameras on-board the twin "Flying Infrared Signature Technology Aircraft (FISTA)". FISTA was about 150 $\mathrm{km}$ north from ARIA and the bulk of meteors are independent records. 
These are probably the result of imperfect corrections for observer perception, observing conditions or other factors that affect visual observations. For the same reason, such features in the profiles from individual locations can not be trusted. In the remainder of this paper, we will concentrate on the gross features of the curves that are confirmed by both video and visual results.

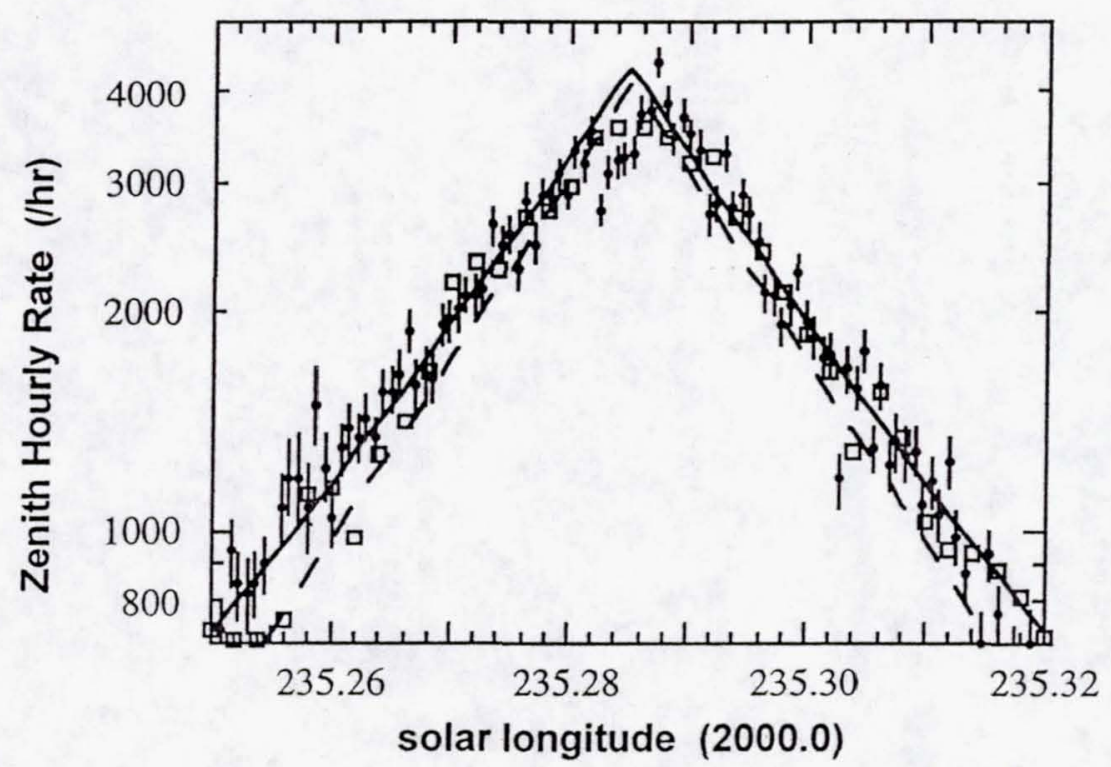

Figure 2. The peak of the 1999 Leonid storm. Solid points are our 1-minute counts (no smoothing applied). Open squares are data from Arlt et al. (1999). The dashed line shows the storm component (main peak), while the solid line is the best fit of all components together.

When plotted on a logarithmic scale, as in Figure 2, it is clear that the slopes of the storm peak are linear and well represented by an exponential equation like (Jenniskens, 1995):

$$
\mathrm{ZHR}=\mathrm{ZHR}_{\max } 10^{-\mathrm{B}\left|\lambda_{\mathrm{o}}-\lambda_{\mathrm{o}}^{\max }\right|}
$$

From a least squares fit, we find $B=24 \pm 2$ per degree solar longitude for $\mathrm{ZHR}$ larger than 700 . A slightly larger $\mathrm{B}=25 \pm 1$ value (and $\mathrm{ZHR}_{\max }$ $=4,100$ per hour) results when a composite of such curves is fitted to the 
1866 and 1966 profiles - Jenniskens, 1995). And that suggests strongly that both components are caused by the same physical processes, with no intrinsic merit to make a distinction between the two components. We expect to be able to verify from the video record that the two components can not be discriminated on ground of the magnitude distribution index, but will take this as a task for a future paper.

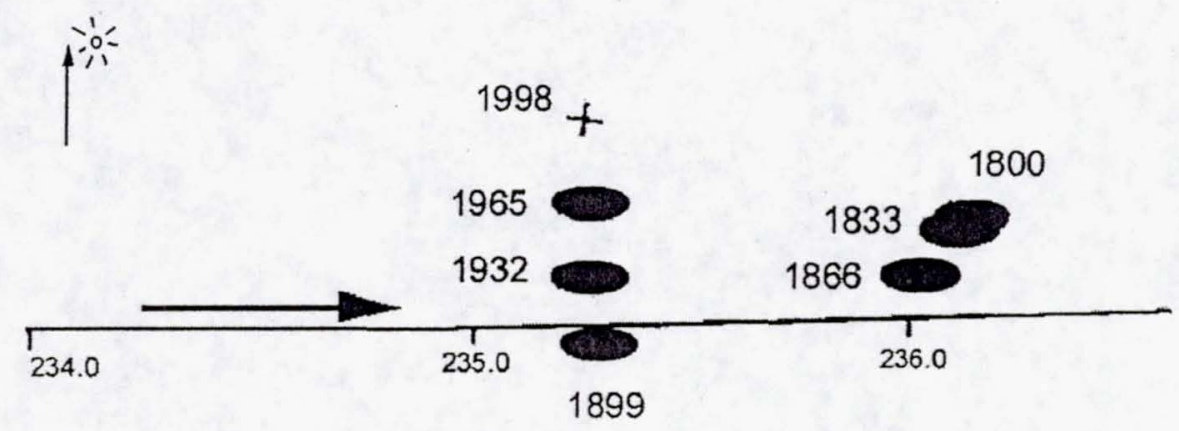

Nov. 17

Nov. 18

Nov. 19

Figure 4. The Earth's path by debris trails ejected at various epochs during the return of 1999. Courtesy: David Asher, Armaugh Observatory.

\subsection{THE 1866 TRAILET}

This material is though to have been ejected in 1899 (Kondrateva and Reznikov, 1985; McNaught and Asher, 1999b; Lyytinen, 1999). In Asher's diagrams of the path of Earth through the meteor shower, reproduced in Figure 4, the Earth approaches dust trails from 1866, 1833 and 1800 shortly after passing the 1899 and 1932 trails. Earlier during the 1998 return, we observed a peak in activity when Earth passed rather far from the calculated center of the 1899 debris trail (Jenniskens, 1999) Hence, we anticipated a second peak of activity just after solar longitude 236.0. Based on observations from Hawaii, Japan and China, Arlt et al. (1999) show this second maximum peak at solar longitude $235.87 \pm$ 0.04 . Leonid MAC observations in the night after the main peak show enhanced rates that appear to trace the declining branch of this component, showing a relatively fast decline (Figure 5). A curve with B $=1.6$ would best fit the descending branch. However, a symmetric curve 


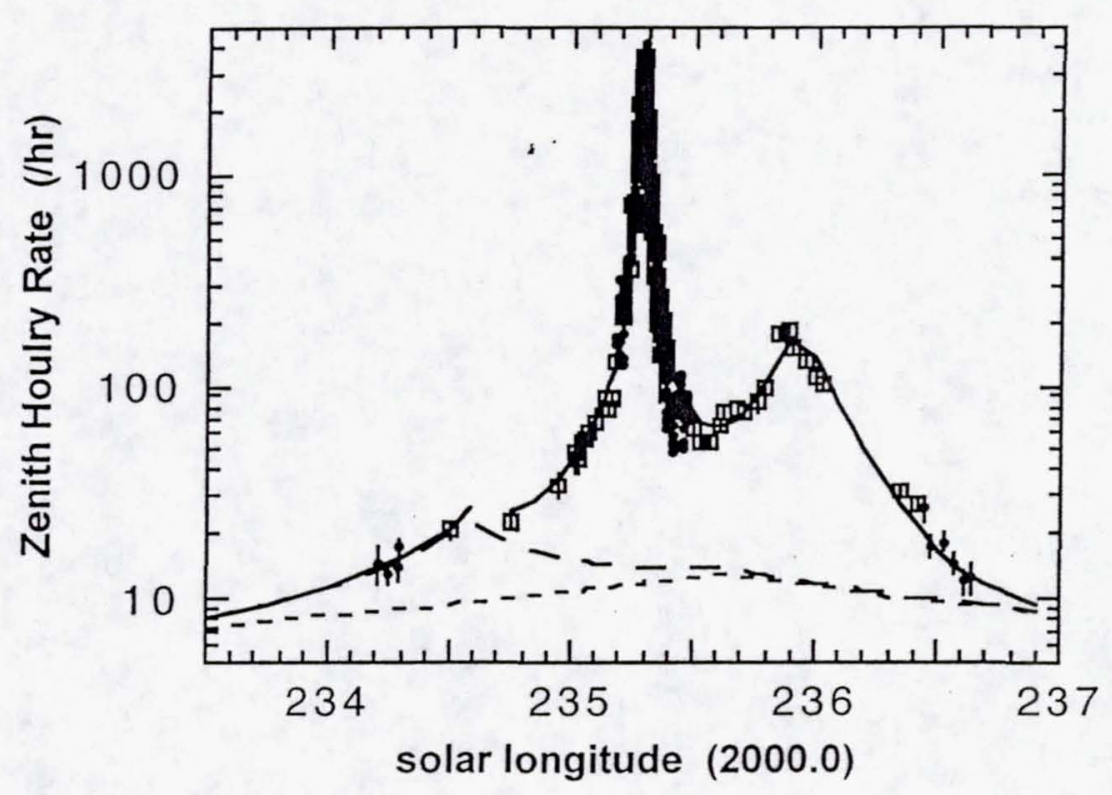

Figure 6. Possible presence of the Leonid Filament component (solid line). Symbols as in Figures 2 and 5.

\subsection{THE LEONID FILAMENT?}

In prior years, another dust component called the Leonid Filament was responsible for fireball showers in all years from 1994 until 1998 (Jenniskens, 1996). Its characteristic feature is the width, with $B=1.1 \pm$ 0.1 in all years, and its low magnitude distribution index. Jenniskens and Betlem (2000) predicted a return of this component at a lower level than in 1998, assuming that the Filament was the accumulation of many years of dust ejecta. Asher (1999), on the other hand, predicted no activity at all if the Filament was due to ejecta of the return of 1333 only.

The 1999 profile does not show a clear broad component that is readily defined as the Leonid Filament. This appears to confirm Asher's prediction. However, Leonid MAC observations in the night prior to the peak night (at solar longitude 234.5) show a significant enhancement of rates above expected levels that may in fact be caused by the Filament. The expected level being a mere extrapolation of the contribution from the annual Leonid shower, the main and background storm peak, and the second 1866/1833 component (Figure 6). This is consistent with few 


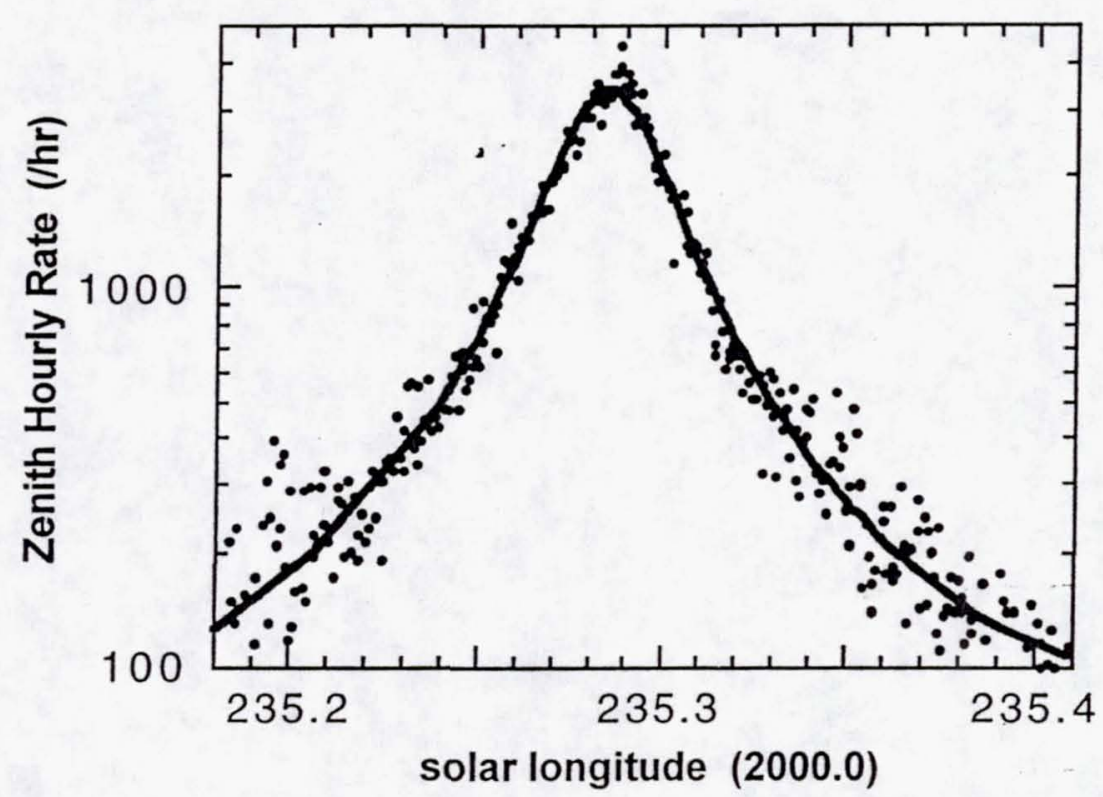

Figure 7. Fit of a Lorentz profile to the meteor storm profile. In order to bring out the small dispersion, error bars are not shown.

We find that data from past meteor storms show a similar good fit (Table I), which implies that each dust trailet itself has a Lorentzian cross section. This condition is necessary to account for the fact that we passed the dust trailets at different distances from the center in 1999, 1966 and 1866. If the dust distribution in a trailet (index " $t$ ") follows a Lorentz function as a function of $r=$ distance from trailet center, then:

$$
\mathrm{ZHR}(\mathrm{r})=\mathrm{ZHR}_{\text {max }}^{\mathrm{t}} \frac{\left(\mathrm{W}_{\mathrm{t}} / 2\right)^{2}}{\mathrm{r}^{2}+\left(\mathrm{W}_{\mathrm{t}} / 2\right)^{2}}
$$

In that case, the cross section is also Lorentzian if we pass the center of the trailet along the Earth's orbit in a direction $X=\lambda_{0}$ (now in $A U$, with roughly $2 \pi \mathrm{AU}=360$ degrees neglecting curvature of the Earth's path) at a distance $\mathrm{Y}=\mathrm{Y}_{\mathrm{o}}$ (measured in a direction perpendicular to Earth's orbit). Because, by substituting $r^{2}=Y_{0}{ }^{2}+\left(X-X_{0}\right)^{2}$ : 
It is not clear, at present, what physical mechanism is responsible for this tail in the distribution. Lorentzian distributions are characteristic for damped oscillators, and perhaps a natural consequence of the orbital evolution in the three body system meteoroid-Sun-Jupiter.

Given the good representation in the case of the 1999 Leonid storm, we applied the Lorentzian fit to other shower components (Table I). We find that this year's shower profile is well represented with three Lorentz curves representing storm, 1866 peak, and Filament (Figure 8).

\section{TABLE I}

\begin{tabular}{|c|c|c|c|c|c|}
\hline Year & From & $\begin{array}{l}\left.\left.\triangle \mathrm{C}-\mathrm{E}^{*}\right) \mathrm{M}_{\mathrm{o}}{ }^{* *}\right) \\
\left.(\mathrm{AU}) \quad{ }^{\circ}\right)\end{array}$ & $\begin{array}{l}\mathrm{W} / 2 \\
(\mathrm{AU})\end{array}$ & $\begin{array}{l}\mathrm{ZHR}_{\max } \\
\left(\mathrm{hr}^{-1}\right)\end{array}$ & $\begin{array}{l}\lambda_{\mathrm{o}}^{\max } \\
\left({ }^{\circ}\right)\end{array}$ \\
\hline \multicolumn{6}{|c|}{ observed: } \\
\hline 1999 & 1899 & $-0.0007 \quad 18.4$ & $0.018 \pm 0.001$ & $3300 \pm 100$ & 235.285 \\
\hline 1998 & 1899 & $(+0.0044) \# 16.6$ & $0.050 \pm 0.005$ & $70 \pm 20$ & (235.28)\# \\
\hline 1966 & 1899 & $-0.0001 \quad 16.6$ & $0.014 \pm 0.0003$ & $15000 \pm 3000$ & 235.166 \\
\hline 1965 & 1899 & +0.00175 .8 & $0.14 \pm 0.04$ & $100 \pm 50$ & 235.40 \\
\hline 1969 & 1932 & +0.000049 .1 & $0.015 \pm 0.003$ & $200 \pm 50$ & 235.265 \\
\hline 1999 & 1866 & +0.001619 .0 & $0.155 \pm 0.015$ & $130 \pm 15$ & 235.95 \\
\hline 1998 & 1866 & +0.004016 .6 & $0.2 \pm 0.1$ & $10 \pm 10$ & 236.0 \\
\hline 1866 & 1733 & -0.00049 .1 & $0.014 \pm 0.004$ & $14000 \pm 2000$ & 233.323 \\
\hline 1867 & 1833 & $\begin{array}{lll}-0.0002 & 19.9\end{array}$ & $0.014 \pm 0.007$ & $5000 \pm 1000$ & 233.411 \\
\hline \multicolumn{6}{|c|}{ predicted: } \\
\hline 2000 & 1866 & +0.000829 .5 & $(0.0011)$ & $(70)$ & $236.28 *)$ \\
\hline 2000 & 1932 & $-0.0012 \quad 29.5$ & $(0.0009)$ & (207) & $235.29 *)$ \\
\hline 2001 & 1866 & +0.000240 .3 & $(0.00053)$ & (72) & $\left.236.46^{*}\right)$ \\
\hline 2002 & 1866 & +0.000051 .1 & $(0.00035)$ & (38) & $\left.236.86^{*}\right)$ \\
\hline 2002 & 1966 & +0.001851 .1 & $(0.0021)$ & (4) & $\left.235.27^{*}\right)$ \\
\hline
\end{tabular}

*) Minimum distance between Earth and Comet orbit, from McNaught \& Asher (1999b)

**) Mean anomaly of trail particles

\#) Large uncertainty because of perturbation by Earth in earlier encounter 
the profiles of 1998, 1965 and the second peak of 1999 are cases of further out. If we plot the width versus the distance to the trailet center (Yo), as calculated by McNaught and Asher (1999b), then we find that Equation 5 (solid line in Figure 9) indeed does fit the result, allowing for at least $\pm 0.0001 \mathrm{AU}$ uncertainty in the calculated trailet positions. The intrinsic width of the dust trailet is calculated at $\mathrm{W}_{\mathrm{t}}=0.00032 \pm 0.00008$ AU.

However, the fit is good only if the calculated trailet pattern (together making up the comet dust trail) is shifted outward by about +0.0003 AU. The curve in Figure 9 should center on zero. We conclude that the Earth crossed about $0.0003 \mathrm{AU}$ deeper into the debris trail ejected in 1899 than predicted. Unfortunately, that means that the Earth will not cross quite as deep into the 1866 epoch trailet in 2001 and 2002.

On top of that are two more factors that influence the peak rate in future years: 1) the rate of decrease of dust density away from the comet for a pristine trailet of 1 revolution, and 2) the decay of dust density with each subsequent revolution.

Regarding the decay of dust density with subsequent revolutions, we assume that the dust density falls off inversely with the number of revolutions $(\mathrm{N})$, which is expected if the spreading is mainly due to differences in orbital period of the particles in the dust trailet. Here, we ignore the fact that the peak of the particle density also shifts progressively along the comet orbit in time. In that case, the peak dust density at a given position after 1 revolution is:

$$
\mathrm{ZHR}_{\max }^{\mathrm{t}}(1 \text { rev. })=\mathrm{ZHR}_{\max }^{\mathrm{t}} \times \mathrm{N}
$$

Figure 10 shows the density of dust in the center of the trailet after one revolution, calculated from the observed peak $\mathrm{ZHR}$ value. This value was corrected to a center-of-trailet value for a Lorentz distribution with adopted $\mathrm{W}_{\mathrm{t}}=0.00032 \mathrm{AU}$ perpendicular to Earth's orbit centered on the trail centers calculated by McNaught and Asher (1999b), and by taking Equation 7 into account. Unfortunately, only one data point (the return of 1969) is available to constrain the slope of the dashed line in Figure 10. All other observations fall in a rather narrow range of mean anomaly. Any error in the 1969 result will bear heavily on the assumed dependence on mean anomaly and the predictions that follow from it. 


\subsection{FUTURE OBSERVATIONS}

The video record of the 1999 Leonid meteor storm is a treasure trove of information that can be further analyzed. Unlike the hybrid visual-video observation technique, such in-depth analysis is time consuming. Some preliminary results are presented in Gural and Jenniskens (2000).

After full analysis of the 1999 shower, the big unknown will still be the dispersion perpendicular to Earth's path and the exact position of the dust trail center. Only the year 2000 encounter can shed light on this. The predicted distances to the trailet centers are small enough to get significant increased rates and recognize the component from other shower components. Also, the distance to the trailet center is not as small as in 2001 and 2002, when we are on the steep slope of the Lorentz profile. Small natural shifts in the trail center can cause great variations in rates that are can not easily be interpreted in terms of the width of the dust trail perpendicular to the Earth's path. On the other hand, while the year 2000 provides a 2 -dimensional picture of what is now only a 1dimensional view of dust trails, the years 2001 and 2002 will provide a three dimensional perspective by providing important clues to how quickly the dust density falls off away from the comet position.

The method described in this paper promises a detailed picture of the dust density in comet dust trailets by combining theory and observations of future Leonid showers. Observations in future years will test the assumptions that go into the model, such as the cylindrical geometry and the position of the trail. Each future encounter will be a strong test for refining the theoretical multi-trailet model of comet dust trails.

\section{Acknowledgements}

We thank ESA's Michael Schmidhuber for assisting in the "flux measurement team" and Morris Jones and Pete Gural who assisted in the visual examination of the FISTA tapes after the flight. The flux measurements in the Leonid MAC 1999 mission were supported by grants from USAF/XOR and the NASA Planetary Astronomy and Suborbital MITM programs. Editorial handling: Noah Brosch. 